\title{
The Wolbachia endosymbiont as an anti-filarial nematode target
}

\author{
Barton E. Slatko • Mark J. Taylor • Jeremy M. Foster
}

Received: 19 December 2009/Accepted: 13 May 2010/Published online: 5 June 2010

(C) The Author(s) 2010. This article is published with open access at Springerlink.com

\begin{abstract}
Human disease caused by parasitic filarial nematodes is a major cause of global morbidity. The parasites are transmitted by arthropod intermediate hosts and are responsible for lymphatic filariasis (elephantiasis) or onchocerciasis (river blindness). Within these filarial parasites are intracellular alpha-proteobacteria, Wolbachia, that were first observed almost 30 years ago. The obligate endosymbiont has been recognized as a target for anti-filarial nematode chemotherapy as evidenced by the loss of worm fertility and viability upon antibiotic treatment in an extensive series of human trials. While current treatments with doxycycline and rifampicin are not practical for widespread use due to the length of required treatments and contraindications, anti-Wolbachia targeting nevertheless appears a promising alternative for filariasis control in situations where current programmatic strategies fail or are unable to be delivered and it provides a superior efficacy for individual therapy. The mechanisms that underlie the symbiotic relationship between Wolbachia and its nematode hosts remain elusive. Comparative genomics, bioinfomatic and experimental analyses have identified a number of potential interactions, which may be drug targets. One candidate is de novo heme biosynthesis, due to its absence in the genome sequence of the host nematode, Brugia malayi, but
\end{abstract}

B. E. Slatko $(\bowtie) \cdot$ J. M. Foster

Molecular Parasitology Division, New England Biolabs,

240 County Road,

Ipswich, MA 01938, USA

e-mail: slatko@neb.com

M. J. Taylor

Filariasis Research Laboratory,

Molecular and Biochemical Parasitology,

Liverpool School of Tropical Medicine,

Pembroke Place,

Liverpool L3 5QA, UK presence in Wolbachia and its potential roles in worm biology. We describe this and several additional candidate targets, as well as our approaches for understanding the nature of the host-symbiont relationship.

Keywords Symbiosis $\cdot$ Wolbachia $\cdot$ Brugia malayi . Filaria . Mutualism · Drug target

\section{Introduction to filariasis}

Filariasis affects over 150 million people in more than 80 countries, with over 1 billion at risk of infection (Molyneux et al. 2003). The causative agents are parasitic filarial nematodes, which induce lymphatic filariasis (LF) and onchocerciasis (river blindness). The species responsible for human lymphatic filariasis are Wuchereria bancrofti, Brugia malayi and B. timori, while human onchocerciasis is caused by the related filarial nematode, Onchocerca volvulus. The life cycle of these parasites requires an arthropod vector; in the case of LF it is transmitted by both anopheline and culicine mosquitoes whereas $O$. volvulus is transmitted by blackflies (Simulium species) (Bogitsh and Cheng 1998; Muller 2002). Adult worms can live for over a decade and are ovoviviparous, releasing millions of fully formed microfilariae (1st stage larvae) into the blood (LF) or the skin (onchocerciasis). Microfilariae are acquired by the insect vector during a blood meal and migrate from the midgut to the thoracic musculature where they develop into third stage larvae. These larvae then migrate to the proboscis from where they can infect another human via the insect bite wound resulting from a subsequent blood feed. The larvae enter the lymphatics (LF) or subcutaneous tissues (onchocerciasis) and molt twice more as they develop into adults. 
Lymphatic filariasis is a disease associated with swellings of the limbs (lymphodema, that can lead to elephantiasis) and scrotal sac (hydrocoele) as a result of damage and dysfunction of the lymphatics. Onchocerciasis (river blindness) presents in sub-cutaneous and deeper tissues as fibrous nodules in which the adult worms reside, skin lesions as a result of inflammation to dead microfilariae, and blindness when microfilariae invade the cornea leading to keratitis, retinal lesions and atrophy of the optic nerve. In general, filarial infections cause little direct mortality but are both disfiguring and debilitating and cause much morbidity and economic loss in endemic countries.

\section{Rediscovery of the Wolbachia endosymbiont of filarial nematodes}

Bacterial-like structures resembling rickettsiales or chlamydiae were first observed in filarial nematodes in the 1970's by electron microscopy (McLaren et al. 1975; Kozek 1977; Kozek and Marroquin 1977), but were then largely overlooked for the next 20 years. The Filarial Genome Project, established in 1994, and funded by the World Health Organization (WHO/Tropical Disease Research/United Nations Development Programme/ World Bank) was one of a small number of projects that simultaneously led to the rediscovery of nematode Wolbachia. Genomic and cDNA sequencing from B. malayi, as part of the WHO initiative, revealed bacterial like sequences which were initially considered cloning contaminants. However, it was soon realized that these sequences were most closely related to the Rickettsiales, and subsequently shown to be from Wolbachia (Williams et al. 2000) which had been taxonomically identified as the endosymbiont within the filarial nematode Dirofilaria immitis (dog heartworm) (Sironi et al. 1995). Wolbachia endosymbionts have now been identified in most filarial nematode species including Brugia spp. and $W$. bancrofti, the causative agents of LF, and in $O$. volvulus, the agent responsible for onchocerciasis (river blindness) (Taylor et al. 2005).

Wolbachia bacteria were first identified in insects almost 100 years ago. In their arthropod hosts (insects, mites, spiders, isopods), Wolbachia are maternally inherited and exhibit a parasitic lifestyle associated with reproductive manipulations such as cytoplasmic incompatibility (spermegg incompatibility), parthenogenesis, feminization and male killing (Werren 1997; Bandi et al. 2001a; Werren et al. 2008). These phenomena are adaptive for Wolbachia and enhance the production of infected females. Wolbachia have been considered as a driving force in evolution likely responsible for reproductive isolation in insects, and potentially useful for sterilization of agricultural pest populations or for reducing insect-borne parasitic disease load (eg.
Dengue fever) (Sinkins and Godfray 2004; Telschow et al. 2005; Sinkins and Gould 2006; Bourtzis 2008; McMeniman et al. 2009; Moreira et al. 2009).

\section{Wolbachia in arthropods and nematodes}

Wolbachia are currently divided into at least seven supergroups and a number of additional lineages (Lo et al. 2002; Casiraghi et al. 2005; Baldo and Werren 2007; Bordenstein et al. 2009). This classification is based mostly upon ribosomal, ftsZ and Wolbachia surface protein (wsp) sequence analysis and a multilocus sequence typing strategy. Among the seven supergroups, two are solely comprised of Wolbachia from nematode hosts (supergroups C and D), while four supergroups (A, B, E, H) only contain Wolbachia from arthropods. Phylogenetic analysis suggests that transfer of Wolbachia, either from arthropods, where they were parasitic, to nematodes, where they became obligate, or vice versa, occurred about 100 mya, after the divergence of nematodes and arthropods (600 mya) (Bandi et al. 1998). An additional supergroup (F) has both arthropod and nematode members, suggestive of a second, much more recent, horizontal transfer between the two host phyla (Casiraghi et al. 2005). However, positioning of the root of the Wolbachia phylogeny and determination of the ancestry of reproductive parasitism and mutualism both appear unresolvable issues with the currently available Wolbachia data sets and the lack of appropriate outgroups (Bordenstein et al. 2009).

It has been proposed that Wolbachia comprise one species, Wolbachia pipientis (Lo et al. 2007), but due to the observations that Wolbachia in filarial nematodes appear to be obligate symbionts whereas in arthropods they are generally reproductive parasites, the one species designation may be only semantic (Pfarr et al. 2007b). In addition, there is considerable phenotypic variation among the arthropod Wolbachia, and Wolbachia genomes are of variable size and their gene orders highly scrambled (Sun et al. 2001; Foster et al. 2005; Werren et al. 2008; Ishmael et al. 2009). However, all Wolbachia share the commonality of being maternally transmitted, although in arthropods there is considerable evidence of lateral transfer among sympatric arthropod species (Heath et al. 1999; Werren et al. 2008).

Wolbachia presence in nematodes appears to be limited to the subfamilies Onchocercinae and Dirofilariinae, within the Onchocercidae family of filarial worms, with the great majority of examined species being infected (Casiraghi et al. 2004). A report of Wolbachia in metastrongylid nematodes of the genus Angiostrongylus (Tsai et al. 2007) appears unreliable and most likely results from sample contamination (Foster et al. 2008). An intriguing situation exists in the plant-parasitic tylenchid nematode Radopho- 
lus similis, from which DNA sequences most closely matching Wolbachia have been identified (Jacob et al. 2008; Haegeman et al. 2009). However, the DNA sequences are highly divergent from the known Wolbachia supergroups, and no gene encoding the diagnostic Wolbachia surface protein could be recovered. Similarly, the antiserum used for immunostaining was a polyclonal raised against whole Wolbachia from arthropods, which would be expected to react with other bacteria. Indeed, the tissue distribution and morphology of the identified bacteria were quite unlike those known for Wolbachia in filarial nematodes. Therefore, the authors leave open the question of whether the bacterium they report might represent a new species and further studies to directly link the DNA sequences with the observed bacterial-like structures are required.

In adult male and female filarial nematodes, Wolbachia are predominantly found within the lateral cords (invaginations of the body wall hypodermis), as well as in ovaries, oocytes and developing embryos in females (Fig. 1). Wolbachia appear to be absent in the male reproductive system. Wolbachia are found within all life-cycle stages, although relative numbers vary between individuals and among different life cycle stages (Fenn and Blaxter 2004a; McGarry et al. 2004). Wolbachia numbers increase dramatically ( $\sim 600$ fold) shortly after infection of the mammalian host giving the highest bacteria / worm ratio of all stages. The numbers are maintained in male worms but continue to

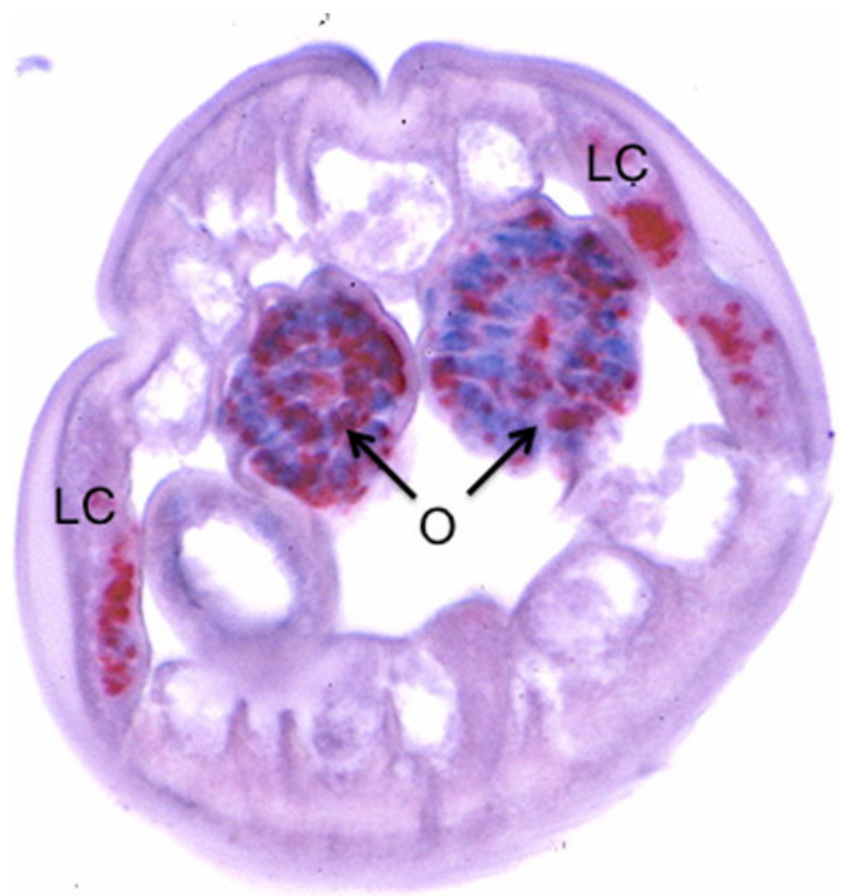

Fig. 1 Distribution of Wolbachia in filarial nematodes. Cross section of adult female B. malayi stained with antibody to Wolbachia surface protein (WSP). Wolbachia (red) are present in the lateral cords (LC) and ovaries $(\mathrm{O})$ increase in female worms as they mature and the ovary and embryonic larvae become infected (McGarry et al. 2004).

As mentioned above, Wolbachia in arthropods are generally considered reproductive parasites. However, several reports in recent years have uncovered mutualistic features of the symbiotic relationship between Wolbachia and certain arthropod hosts. These include beneficial effects on arthropod fecundity, longevity as well as protection from viral infection and metabolic provisioning during periods of nutritional stress (Dedeine et al. 2003; Alexandrov et al. 2007; Dong et al. 2007; Panteleev et al. 2007; Hedges et al. 2008; Brownlie et al. 2009). Importantly, the supergroup F Wolbachia endosymbiont present in the bedbug Cimex lectularius was recently shown to be a bacteriocyte-associated obligate nutritional mutualist. Removal of the bacterium leads to retarded growth and sterility (Hosokawa et al. 2010). The former distinction between reproductive parasitism in arthropods and obligate mutualism in filarial nematodes appears to becoming increasingly nebulous.

The generally accepted view of the association between Wolbachia and their filarial nematode hosts is one of obligate mutualism. This is based predominantly on i) the congruent phylogenies of the endosymbiont and their nematode hosts indicative of long, stable co-evolution, implying a reciprocal dependence, ii) observations that all individuals of infected species contain Wolbachia, and iii) the detrimental effects of antibiotics such as doxycycline on worm fertility, development and viability (Taylor et al. 2005). These antibiotics have adverse effects on filarial nematodes that harbor Wolbachia, but not on Wolbachiafree filaria, confirming that the anti-filarial properties are mediated through Wolbachia.

\section{Wolbachia as a target for filariasis control}

The principle of treating filarial disease through antibiotic treatment is well established by multiple studies reporting anti-filarial effects of antibiotics such as doxycycline or rifampicin on worms maintained in vitro, or in vivo, including several clinical trials in humans (Taylor et al. 2005; Bazzocchi et al. 2008; Hoerauf et al. 2008; Specht et al. 2008; Supali et al. 2008; Coulibaly et al. 2009; Mand et al. 2009; Wanji et al. 2009). Adverse effects include inhibition of nematode embryogenesis, infertility, inhibition of larval development, stunting of adult worms and macrofilaricidal (adult worm killing) activity. These effects correlate with clearance of Wolbachia from worm tissues and indicate that the bacterium provides essential function to the host nematode that allows correct development and viability. Furthermore, it has not been possible to produce viable worms that are totally "cured" of their Wolbachia. 
Previous strategies for treatment of filarial infections have had limited effect upon adult worms, which can persist in humans for 10 years or more. Adults mate and give rise to microfilaria within the mammalian host enabling continuation of the infection cycle by arthropod uptake. Macrofilaricides are therefore of practical importance. Control programs rely on sustained delivery of antiparasitic drugs, such as DEC (diethylcarbamazine), albendazole, and ivermectin, which have been the mainline drugs of choice for filariasis control. However, these drugs are not effective adulticides and repeated community-wide doses are required to suppress microfilarial production and reduce transmission. In addition, the possibility of drug resistance, as observed with intestinal helminths in animals, is a growing concern (Bourguinat et al. 2006; Eng et al. 2006; Bourguinat et al. 2007; Osei-Atweneboana et al. 2007). No new drugs against filariae with good efficacy and safety profile have been developed in over 20 years, and there is a need for drugs that permanently sterilize or kill adult worms.

The antibiotic approach of targeting Wolbachia endobacteria for filariasis control has been successfully demonstrated in humans through drug trials with doxycycline or rifampicin. Long-term sterilization, as well as adult worm killing of $O$. volvulus, and lymphatic filarial nematodes $(B$. malayi and $W$. bancrofti) occurs using typically three to eight-week courses of antibiotic (Hoerauf et al. 2001; Taylor et al. 2005; Hoerauf et al. 2008; Specht et al. 2008; Supali et al. 2008; Mand et al. 2009; Turner et al. 2010). However, the antibiotic treatments used in these studies are of long duration and are contra-indicated for children under eight years old and for pregnant or breastfeeding women. Discovery of additional drugs active against Wolbachia without these contraindications is imperative. An analysis of Wolbachia genome sequences and symbiotic mechanisms is predicted to contribute to the development of new candidate drug targets.

\section{Genome sequencing of the B. malayi Wolbachia endosymbiont}

Careful analysis of genomic sequences is expected to facilitate identification of potential targets and aid elucidation of the symbiotic mechanisms underlying the Wolbachianematode association, which in turn will also contribute to the target identification approach. To date the only completed Wolbachia genome from any filarial nematode is that from B. malayi (Foster et al. 2005). The nuclear genome sequence of $B$. malayi is also available (Ghedin et al. 2007) permitting comparisons of, for example, host and endosymbiont metabolism to highlight likely cases of metabolic provisioning. Very recently, large DNA contigs from genome sequencing projects of $O$. volvulus and $W$. bancrofti, both of which contain Wolbachia, and Loa loa, a filarial nematode free of Wolbachia, have been deposited in the NCBI database. These data allow more extensive comparisons of filarial and endosymbiont genomes and cross-species comparisons that can include Wolbachia-free filarial worms.

Genome sequencing of the Wolbachia endosymbiont from $B$. malayi revealed a $66 \% \mathrm{~A}+\mathrm{T}$ rich genome of about $1.1 \mathrm{Mb}$, potentially encoding 806 open reading frames for functional protein translation (Foster et al. 2005). The genome contains one copy of each of the ribosomal genes, which are not present in an operon. The genome has an extremely low density of functional genes compared to most other bacteria, and has undergone considerable gene loss in many metabolic pathways. There is a loss of transcriptional regulators, suggesting constitutive expression of most proteins. While most signal transduction systems are also depleted, stress response and heat shock proteins are present. Only a few amino acids can be synthesized while the remainder need to be imported by use of a significant set of proteases and peptidases and degradation/import pathways. Complete pathways for purine and pyrimidine de novo synthesis are present, in contrast to the host worms lack of these pathways. Fatty acid biosynthesis and phospholipid biosynthesis can occur, as can FAD and riboflavin biosynthesis. Biosynthesis of these latter two compounds is absent in B. malayi, which must acquire them from extraneous sources or perhaps from their endosymbiont. Interestingly, oral supplementation of B vitamins to bedbugs depleted of their nutritional mutualistic Wolbachia (supergroup F) rescues their growth and sterility defects (Hosokawa et al. 2010). Wolbachia has incomplete pathways for biosynthesis of certain vitamins and cofactors such as NAD, biotin, lipoic acid, ubiquinone, folate, pyridoxal phosphate, and Coenzyme A, making them dependent on external sources, such as from the host. Heme biosynthesis can occur in Wolbachia (see below), but not in the host, as the requisite genes are missing. Glutathione biosynthesis can occur, and may facilitate oxidative stress reduction, phosphate uptake or be provisioned to the nematode host.

Glycolysis is probably not operational in Wolbachia since two enzymes (6-phosphofructokinase and pyruvate kinase) are absent but replaced by the gluconeogenic enzyme, fructose-1,6-bisphosphatase and by pyruvatephosphate dikinase (PPDK) which is also predominantly a gluconeogenic enzyme in bacteria. [Of note, PPDK represents an attractive target since it is absent in mammals, which use pyruvate kinase instead]. Thus the glycolytic pathway appears to operate in the reverse direction with a gluconeogenic function. In this scenario, amino acids supplied by the host or as proteolysis products can be used to enter the functional citric acid cycle at several steps, leading to acetyl CoA and then to glyceraldehyde-3- 
phosphate (G-3-P) via gluconeogenesis. This can then be metabolized by the nonoxidative pentose phosphate pathway to yield the pentoses necessary for biosynthesis of nucleotides and cofactors such as FAD and riboflavin. A lack of sugar transporters and kinases and a lack of lactate dehydrogenases also suggest that these compounds are not utilized for growth.

Functional Type IV secretion genes were identified in the genome sequence, and are generally required for maintenance of endosymbionts within their hosts, and thus likely play a role in the intracellular adaptation. Several ankyrin-repeat-containing proteins (Anks) are predicted and have potential functions that include connection of symbiont cell membranes to the cytoskeleton. In other intracellular bacteria, Anks are frequently secreted into the host cell via the Type IV secretion system and act as effector proteins important for infection of the host cell (Pan et al. 2008). For example, AnkA from the closely related rickettsial organism Anaplasma phagocytophila is critical for infection of the eukaryotic host cell via activation of the host Abl-1 signaling pathway (Lin et al. 2007). AnkA binds chromatin and nuclear proteins of the host cell where it is believed to modulate cell cycle control (Caturegli et al. 2000). Another interesting protein is a member of the WASP family, which can regulate the formation of actin filaments. The genomic sequence thus reveals molecules that might be involved in the symbiotic relationship and a number of potential targets in Wolbachia biochemistry.

\section{Other genes of interest and approaches towards target identification}

The evolutionary distance of Wolbachia from mammals is far greater than that of nematodes, affording opportunities for treating filarial infections by specifically targeting the endosymbiont. A number of targets have been identified by genomic analysis, directed bioinformatic-based discovery and experimental approaches and include membrane proteins, ankyrins, lipoprotein biosynthesis, enzymes of lipid II biosynthesis, heme biosynthesis and the glycolytic enzymes PPDK and cofactor-independent phosphoglycerate mutase among others (Pfarr et al. 2007a; Ghedin et al. 2008; Raverdy et al. 2008; Foster et al. 2009; Henrichfreise et al. 2009; Holman et al. 2009; Wu et al. 2009; Johnston et al. manuscript submitted).

The A-WOL consortium is also screening compounds and small molecules from a variety of sources, including novel tetracyclines and single agents, and combinations of FDA approved drugs, focused anti-bacterial libraries and larger diversity-based natural product and synthetic compound libraries in whole cell-based assays, nematode in vitro assays and in vivo filarial models. The principal goal is the establishment of anti-symbiotic chemotherapy directed against Wolbachia, compatible with MDA programs for human filariasis and optimizing individual therapy and community based delivery for a more restricted use. For example, in the event of the emergence of drug-resistance to existing treatments in limited populations or as an alternative for individual poor-responders. Several potential "hits" have been identified and are being prioritized for further screening (http://A-WOL.com).

We have initiated interactome studies to aid in the identification of potential functional and interacting molecules. The interactome strategy utilizes Wolbachia proteins expressed as GST fusions, which are then bound to glutathione columns. B. malayi protein extracts are passed though the column and bound "interactors" eluted for subsequent identification by mass spectrometry. We and others are performing microarray experiments to examine filarial and/ or Wolbachia gene expression in different life-cycle stages, during filarial larval molting, and under different doses of doxycycline to help identify changes in expression level in response to antibiotic stress (Ghedin et al. 2009; Strubing et al. 2010) (B. Wu, J. Foster, B. Slatko, unpublished results). In addition, proteomic approaches are beginning to be deployed to detect Wolbachia proteins from different filarial lifecycle stages, for example amongst the excreted / secreted proteome of worms maintained in vitro (Bennuru et al. 2009).

\section{Heme biosynthesis: a novel candidate Wolbachia drug target}

Genomic comparison of Wolbachia (Foster et al. 2005) and its B. malayi nematode host (Ghedin et al. 2007) may provide insight into the metabolic contributions to the mutualistic symbiotic relationship. One such pathway, mentioned earlier, is heme biosynthesis. Heme is an essential cofactor for many proteins including cytochromes, hemoglobins, peroxidases, and catalases that are involved in a wide range of critical biological processes, including oxidative metabolism and electron transport. All but one of the heme biosynthetic genes are present in the Wolbachia genome, with the missing step being protoporphyrinogenIX oxidase, a step unidentified in many heme-producing gram-negative bacteria. As with other nematodes, the heme biosynthetic pathway is absent in B. malayi (Rao et al. 2005; Ghedin et al. 2007), requiring acquisition of heme or intermediates from Wolbachia or salvage from their surroundings. Interestingly, Wolbachia have been shown to enhance fecundity of Drosophila melanogaster reared on an iron-deficient diet (Brownlie et al. 2009). Elimination of Wolbachia following antibiotic treatment affects a number of processes in the nematode, which may be related to heme deprivation. This is further suggested by the use of 
two heme biosynthesis inhibitors, succinyl acetone (SA) and N-methyl mesoporphyrin (NMMP), which target aminolevulinic acid dehydratase (ALAD) and FC, respectively. Treatments with these show effects upon adult and microfilarial worm motility (Wu et al. 2009). Furthermore, mitochondrial genes for heme-dependent respiratory chain complexes are up-regulated after depletion of Wolbachia from filarial nematodes, but not after antibiotic treatment of a naturally endosymbiont-free species (Strubing et al. 2010).

E.coli heme deficient mutant strains (hemB, hemD and hemH) have been used to perform functional complementation tests with the corresponding cloned Wolbachia enzymes. These assays complement those described above and verify the functionality of the clones tested. For example, an E. coli hem $B$ mutant transformed with a plasmid with no insert DNA fails to grow on LB plates, unless hemin is added to the media. Transformations of the E. coli hemB mutant strain with a functional cloned Wolbachia ALAD insert results in colony growth on LB plates without hemin addition, similar to wild type $E$. coli growth. Similar complementation was observed for $E$. coli hemD and hemH mutants using Wolbachia uroporphyrinogen III synthase (UROS) and Wolbachia FC, respectively (Wu et al. 2009). Wolbachia ALAD and FC only share $22-34 \%$ identities $(29-53 \%$ similarities) with their human homologues and thus recombinant proteins were assayed for their differential biochemical properties. In both cases, there were significant differences in the biochemistry between the Wolbachia and human homologues, making the Wolbachia heme biosynthetic pathway of interest for drug targeting (Wu et al. 2009). Recombinant proteins have been purified, assayed for activity and submitted for aptamer/small molecule screening as part of the A-WOL consortium. RNA aptamers have been identified that selectively bind the Wolbachia proteins but not their human orthologs, and small molecule screening is underway to identify compounds that compete off the bound aptamers together with target high-throughput screening against large diversity based drug libraries.

\section{The ferrochelatase gene of $B$. malayi: a functional lateral gene transfer}

A peculiar feature of the phylum Nematoda is the apparent absence of de novo heme biosynthesis (Rao et al. 2005), although the genomic sequence of $B$. malayi revealed the presence of a potential ferrochelatase (FC) gene (Ghedin et al. 2007), catalyzing the last step in the biosynthetic pathway. It appears that filarial nematodes may be unique among nematodes in possessing this terminal step of heme biosynthesis, as a consequence of a lateral gene transfer (LGT) event. Full-length FC cDNA sequences from $B$. malayi, O. volvulus, D. immitis and the Wolbachia-free filarial worm Acanthocheilonema viteae have been obtained from adult worm RNA preparations via PCR/RT-PCR/ RACE analyses and the predicted protein sequences are most similar to $\alpha$-proteobacterial FCs. (B. Wu and B. Slatko, unpublished results). Surprisingly, phylogenetic analysis shows that the filarial FC genes did not originate from Wolbachia, which possesses a fully functional heme biosynthetic pathway, but most likely from an $\alpha$ - proteobacterium belonging to the order Rhizobiales.

The full-length $B$. malayi FC gene contains 9 exons spanning across $\mathrm{a} \sim 4 \mathrm{~kb}$ genomic region. The first exon encodes a 36 aa N-terminal pre-sequence, which is generally not present in bacterial FCs and contains a putative mitochondriontargeting peptide, as predicted by TargetP. The signature residues required for catalysis are strictly conserved, suggesting the predicted filarial FC proteins are functional enzymes and this was confirmed by both $E$. coli complementation tests and in vitro enzyme assays. Heterologous expression in $C$. elegans demonstrated that the $\mathrm{N}$-terminal pre-sequence is required for mitochondrial targeting. In all non-plant eukaryotes possessing the heme biosynthetic pathway, FCs are mitochondrial-resident enzymes, so this result agrees with a role for the filarial FCs in heme biosynthesis. Wild type $C$. elegans larvae are required to salvage heme for development into adulthood due to total absence of heme biosynthetic capability. However, $B m \mathrm{Fc}$ transgenic $C$. elegans larvae can utilize the heme precursor protoporphyrin IX, further demonstrating that filarial FCs are fully functional enzymes. Ex vivo A. viteae and Wolbachia-depleted $B$. malayi motility and viability assays in the presence of the FC specific inhibitor NMMP also suggest that this filarial FC might be essential for parasite survival (B. Wu, B. Slatko, L. Ford and M. Taylor unpublished results). Since A. viteae lacks the Wolbachia endosymbiont, this observation implies that the anti-filarial effect of NNMP is due to inhibition of the FC of Rhizobial origin encoded by the nematode nuclear genome. In Wolbachia-dependent filarial worms, the endosymbiont FC is a second target for NNMP. This is the first case of a functional lateral gene transfer event in animal parasitic nematodes and reinforces our suggestions that heme biosynthesis is a targetable option for anti-filarial chemotherapy.

\section{Do lateral gene transfers play a role in Wolbachia evolution and symbiosis?}

Wolbachia DNA has been identified in the nuclear genomes of endosymbiont-dependent parasites (Fenn et al. 2006; Hotopp et al. 2007). In these cases, the inserted genes and gene fragments are all degenerated and incapable of encoding protein that would match the corresponding protein encoded by the Wolbachia genome. This now appears to also be the case for certain filarial species that 
are naturally endosymbiont-free, indicating that the ancestors of these species contained Wolbachia. "NextGen" 454 DNA sequencing of two distantly related Wolbachia-free filarial species, $A$. viteae and $O$. flexuosa identified 45 and 92 genomic contigs, containing 49 and 114 Wolbachia gene fragments, respectively (McNulty et al. 2010). Many of these sequences show insertions and/or truncations relative to the functional homolog, non-synonymous nucleotide substitutions, frameshift mutations and premature stop codons suggestive of a lack of selection pressure for maintaining their functionality. However, only a small part of the genome has been scanned by the low coverage ( $\sim 2$ to $3 \mathrm{X})$ sequence survey and further sequencing will be required to determine if any functional transferred sequences exist. Examination of the DNA contigs deposited in NCBI from genomic sequencing of a third Wolbachia-free filarial nematode, Loa loa, indicates past infection with Wolbachia in this filarial species also.

The mechanism by which the Wolbachia DNA entered the host nematode genomes is unknown. It may have involved the Wolbachia Type IV secretion system, which is capable of shuttling proteins and nucleoprotein complexes across membranes and known to facilitate gene transfer (Juhas et al. 2008). After translocation, DNA may have been inserted into the host genome by transposable elements or using Wolbachia recombination systems. Remnants of retrotransposons and large poly(A) stretches found in some of the sequences (McNulty et al. 2010) suggest that some may be processed pseudogenes derived from mature mRNAs (Podlaha and Zhang 2009). If this is correct, a Wolbachia homolog must have been expressed from the filarial genome at some time. Of interest is that approximately half of the degenerate gene fragments appear to be transcribed. By qRTPCR mRNA expression was detected for 30 A. viteae and 56 $O$. flexuosa Wolbachia-like sequences and in situ hybridization of two of these sequences showed them to be specifically expressed in developing embryos and testes. The transcript levels of these Wolbachia insertions relative to functional nematode genes were not determined. However, Wolbachia gene sequences inserted into the Drosophila ananassae nuclear genome were shown to be transcribed at levels $10^{4}$ to $10^{7}$ less than the fly actin gene, but since there is no cut-off for biologically relevant transcription, which can vary widely both between genes and temporospatially, the significance of these transcribed Wolbachia genes remains unresolved (Hotopp et al. 2007). How these endosymbiont-free filarial species were able to loose their Wolbachia while in other species Wolbachia presence appears obligatory remains unknown. Are there additional unidentified but functional Wolbachia sequences that were transferred that could be the basis for allowing the "obligate" Wolbachia to be lost? Are any of the sequences involved in the symbiotic relationship?

\section{Is the symbiotic relationship between Wolbachia and filarial nematodes exclusively an obligate mutualism?}

As mentioned previously (Section 3), the accepted view of the symbiotic association between Wolbachia and their filarial nematode hosts is one of obligate mutualism. However, several lines of evidence call into question this generic designation, which may be an oversimplification for the group as a whole. For example, as discussed earlier, several filarial nematodes do not contain Wolbachia and some of these endosymbiont-free species, such as $O$. flexuosa and Litomosoides yutajensis, belong to genera that are otherwise infected with Wolbachia.

The absence of Wolbachia in certain filariae could reflect preservation of an ancestral condition or, alternatively, secondary loss of endosymbiont. Secondary loss of endosymbiont appears the most plausible explanation since in three Wolbachia-free species (O. flexuosa, A. viteae and $L$. loa), fragments of Wolbachia genes have now been identified in the nematode nuclear genomes by low coverage genomic sequencing, as discussed above, implying their ancestors were once infected. These multiple independent losses of Wolbachia are difficult to reconcile with an obligatory symbiosis (Fenn and Blaxter 2004b). It might be argued that Wolbachia genes essential for filarial nematode survival have been incorporated into the genomes of the endosymbiont-free species by LGT, allowing subsequent loss of Wolbachia, but to date only degenerated, short Wolbachia gene fragments have been identified in such genomes. More extensive genomic sequencing of various Wolbachia-free filariae is required to fully address this topic.

Observations on Mansonella perstans and certain filarial nematodes infecting wildlife suggest a strict obligatory mutualism with Wolbachia may not occur in some of these species. Wolbachia-infected and Wolbachia-uninfected populations of $M$. perstans have been identified in different regions of Africa. (Keiser et al. 2008). This is in contrast to the evidence from other Wolbachia-infected species in which all individual worms and populations contain Wolbachia as predicted for an obligatory association. The endosymbiont of M. perstans, when present, clusters with Wolbachia supergroup $\mathrm{F}$, which predominantly contains arthropod Wolbachia, including the obligate mutualistic endosymbiont of bedbugs (Casiraghi et al. 2005; Hosokawa et al. 2010). It appears that a relatively recent transfer of an arthropod Wolbachia into some populations of $M$. perstans has occurred. Doxycycline treatment of $M$. perstans infections in an area where the nematodes carry Wolbachia resulted in a decreased microfilaremia suggesting that the endosymbiont has assumed some functions necessary for worm fecundity (Coulibaly et al. 2009). Members of the genus Cercopithifilaria generally have no Wolbachia but C. japonica from 
bears have been found to be infected (Bain et al. 2008). The endosymbiont is also a supergroup F Wolbachia, like that from Mansonella, and presumably was also acquired from an arthropod. It may be relevant that the Wolbachia within these filarial species cluster with arthropod Wolbachia in supergroup F, and that a relatively recent transfer of Wolbachia between these arthropod and nematode species is indicated (Casiraghi et al. 2005). The apparent lack of complete saturation of Wolbachia in these few nematode species may therefore reflect a more recent arthropod origin where parasitism generally prevails (Werren 1997; Clark 2007). Conceivably, Wolbachia do not form an obligate mutualism in those filarial species that appear to contain both infected and uninfected worms. However, antibiotic treatment leads to reduced microfilaremia in human infections with $M$. perstans in an area where the nematode population harbors Wolbachia (Coulibaly et al. 2009), indicative of mutualism.

It is difficult to reconcile anything other than an obligatory mutualism with the unequivocal anti-filarial effects of antibiotics on filarial nematodes harboring Wolbachia. One alternative possibility is that filarial nematodes have become genetically addicted to Wolbachia and have evolved in response to bacterial presence in their tissues. Some evidence for this scenario is provided by observation of an apparent mutualism between Wolbachia and the parasitic wasp, Asobara tabida. In this system, antibiotic curing of Wolbachia results in abnormal development of the ovaries (Dedeine et al. 2001). The presence of Wolbachia in ovarian tissues of A. tabida dampens apoptotic processes so removal of the bacteria with antibiotics leads to increased death of ovarian cells through apoptosis (Pannebakker et al. 2007). Although this particular bacteria-arthropod association has features of mutualism (Dedeine et al. 2001; Pannebakker et al. 2007), it has also been interpreted as a case of genetic addiction where the wasp has evolved in response to Wolbachia presence (Werren et al. 2008). However, the outward manifestations of such a genetic addiction and mutualism appear to be the same. A second possibility to account for the anti-filarial effects of antibiotics that has been discussed previously is that dying or dead bacteria are themselves toxic to the surrounding nematode tissues (Hoerauf et al. 1999; Bandi et al. 2001b; Taylor 2002; Taylor et al. 2005). However, the authors of these reports note that clearance of Wolbachia from filarial tissues precedes detrimental effects on worm development by weeks and on viability by several months or even years. Furthermore, the mode of action of doxycycline is only bacteriostatic so there is no sudden release of bacterial components; rather just a natural attrition of the bacteria which fail to divide to maintain their population.

Despite uncertainties about whether the designation of obligate mutualism universally fits all Wolbachia-filarial nematode associations, the available evidence supports this form of symbiosis in the causative agents of human lymphatic filariasis and onchocerciasis. Anti-Wolbachia strategies are clearly feasible (Wanji et al. 2009) and offer exciting prospects for the development of new approaches towards filarial disease control.

\section{Future directions / concluding remarks}

Unlike many bacteria-invertebrate symbiotic associations where analysis of the genome sequences has exposed the metabolic provisioning between partners (Shigenobu et al. 2000; Moran et al. 2003; Wu et al. 2006), the precise nature of the association between Wolbachia and their filarial nematode hosts remains enigmatic. Even our traditional definition of Wolbachia as obligate mutualist in nematodes and reproductive parasites in arthropods is becoming less clear as more of these symbiotic relationships are investigated. Analysis of genome sequences from $B$. malayi and its Wolbachia endosymbiont has predicted candidate processes which may underlie the symbiotic relationship (Foster et al. 2005; Ghedin et al. 2007), but verification of these hypotheses requires detailed investigation. The study of Wolbachia-filarial nematode symbiosis stands at an exciting threshold as more genomic sequences are emerging and post-genomic technologies such as microarray analysis, proteomics, metabolomics and interactome studies are being developed to supplement biochemical and cell biology approaches. It is anticipated that in the coming years these investigations will unravel the determinants of the symbiotic relationship. Such knowledge should direct new avenues for developing therapies to treat the debilitating infections caused by filarial nematodes that remain a scourge across much of the tropical and sub-tropical world.

Acknowledgements We thank Dr. Donald Comb and New England Biolabs for continued interest and support of our work. This work was also supported by a grant from the Bill and Melinda Gates Foundation to the Liverpool School of Tropical Medicine as part of the A-WOL consortium.

Open Access This article is distributed under the terms of the Creative Commons Attribution Noncommercial License which permits any noncommercial use, distribution, and reproduction in any medium, provided the original author(s) and source are credited.

\section{References}

Alexandrov ID, Alexandrova MV, Goryacheva II, Rochina NV, Shaikevich EV, Zakharov IA (2007) Removing endosymbiotic Wolbachia specifically decreases lifespan of females and competitiveness in a laboratory strain of Drosophila melanogaster. Russ J Genet 43:1147-1152

Bain O, Casiraghi M, Martin C, Uni S (2008) The nematoda Filarioidea: critical analysis linking molecular and traditional approaches. Parasite 15:342-348 
Baldo L, Werren JH (2007) Revisiting Wolbachia supergroup typing based on WSP: spurious lineages and discordance with MLST. Curr Microbiol 55:81-87

Bandi C, Anderson TJ, Genchi C, Blaxter ML (1998) Phylogeny of Wolbachia in filarial nematodes. Proc Biol Sci 265:2407-2413

Bandi C, Dunn AM, Hurst GD, Rigaud T (2001a) Inherited microorganisms, sex-specific virulence and reproductive parasitism. Trends Parasitol 17:88-94

Bandi C, Trees AJ, Brattig NW (2001b) Wolbachia in filarial nematodes: evolutionary aspects and implications for the pathogenesis and treatment of filarial diseases. Vet Parasitol 98:215238

Bazzocchi C, Mortarino M, Grandi G, Kramer LH, Genchi C, Bandi C, Genchi M, Sacchi L, McCall JW (2008) Combined ivermectin and doxycycline treatment has microfilaricidal and adulticidal activity against Dirofilaria immitis in experimentally infected dogs. Int J Parasitol 38:1401-1410

Bennuru S, Semnani R, Meng Z, Ribeiro JM, Veenstra TD, Nutman TB (2009) Brugia malayi excreted/secreted proteins at the host/ parasite interface: stage- and gender-specific proteomic profiling. PLoS Negl Trop Dis 3:e410

Bogitsh BJ, Cheng TC (1998) Human parasitology. Academic, San Diego

Bordenstein SR, Paraskevopoulos C, Hotopp JC, Sapountzis P, Lo N, Bandi C, Tettelin H, Werren JH, Bourtzis K (2009) Parasitism and mutualism in Wolbachia: what the phylogenomic trees can and cannot say. Mol Biol Evol 26:231-241

Bourguinat C, Pion SD, Kamgno J, Gardon J, Gardon-Wendel N, Duke BO, Prichard RK, Boussinesq M (2006) Genetic polymorphism of the beta-tubulin gene of Onchocerca volvulus in ivermectin naive patients from Cameroon, and its relationship with fertility of the worms. Parasitology 132:255-262

Bourguinat C, Pion SD, Kamgno J, Gardon J, Duke BO, Boussinesq M, Prichard RK (2007) Genetic selection of low fertile Onchocerca volvulus by ivermectin treatment. PLoS Negl Trop Dis 1:e72

Bourtzis K (2008) Wolbachia-based technologies for insect pest population control. Adv Exp Med Biol 627:104-113

Brownlie JC, Cass BN, Riegler M, Witsenburg JJ, Iturbe-Ormaetxe I, McGraw EA, O’Neill SL (2009) Evidence for metabolic provisioning by a common invertebrate endosymbiont, Wolbachia pipientis, during periods of nutritional stress. PLoS Pathog 5:e1000368

Casiraghi M, Bain O, Guerrero R, Martin C, Pocacqua V, Gardner SL, Franceschi A, Bandi C (2004) Mapping the presence of Wolbachia pipientis on the phylogeny of filarial nematodes: evidence for symbiont loss during evolution. Int $\mathrm{J}$ Parasitol 34:191-203

Casiraghi M, Bordenstein SR, Baldo L, Lo N, Beninati T, Wernegreen JJ, Werren JH, Bandi C (2005) Phylogeny of Wolbachia pipientis based on gltA, groEL and ftsZ gene sequences: clustering of arthropod and nematode symbionts in the F supergroup, and evidence for further diversity in the Wolbachia tree. Microbiology 151:4015-4022

Caturegli P, Asanovich KM, Walls JJ, Bakken JS, Madigan JE, Popov VL, Dumler JS (2000) ankA: an Ehrlichia phagocytophila group gene encoding a cytoplasmic protein antigen with ankyrin repeats. Infect Immun 68:5277-5283

Clark ME (2007) Wolbachia symbiosis in arthropods. In: Hoerauf A, Rao RU (eds) Wolbachia: a bug's life in another bug. Karger, Basel, pp 90-123

Coulibaly YI, Dembele B, Diallo AA, Lipner EM, Doumbia SS, Coulibaly SY, Konate S, Diallo DA, Yalcouye D, Kubofcik J, Doumbo OK, Traore AK, Keita AD, Fay MP, Traore SF, Nutman $\mathrm{TB}$, Klion AD (2009) A randomized trial of doxycycline for Mansonella perstans infection. N Engl J Med 361:1448-1458
Dedeine F, Vavre F, Fleury F, Loppin B, Hochberg ME, Bouletreau M (2001) Removing symbiotic Wolbachia bacteria specifically inhibits oogenesis in a parasitic wasp. Proc Natl Acad Sci U S A 98:6247-6252

Dedeine F, Bandi C, Bouletreau M, Kramer LH (2003) Insights into Wolbachia obligatory symbiosis. In: Bourtzis K, Miller TA (eds) Insect Symbiosis. CRC Press, Boca Raton, pp 267-282

Dong P, Wang JJ, Hu F, Jia FX (2007) Influence of Wolbachia infection on the fitness of the stored-product pest Liposcelis tricolor (Psocoptera: Liposcelididae). J Econ Entomol 100:1476-1481

Eng JK, Blackhall WJ, Osei-Atweneboana MY, Bourguinat C, Galazzo D, Beech RN, Unnasch TR, Awadzi K, Lubega GW, Prichard RK (2006) Ivermectin selection on beta-tubulin: evidence in Onchocerca volvulus and Haemonchus contortus. Mol Biochem Parasitol 150:229-235

Fenn K, Blaxter M (2004a) Quantification of Wolbachia bacteria in Brugia malayi through the nematode lifecycle. Mol Biochem Parasitol 137:361-364

Fenn K, Blaxter M (2004b) Are filarial nematode Wolbachia obligate mutualist symbionts? Trends Ecol Evol 19:163-166

Fenn K, Conlon C, Jones M, Quail MA, Holroyd NE, Parkhill J, Blaxter M (2006) Phylogenetic relationships of the Wolbachia of nematodes and arthropods. PLoS Pathog 2:e94

Foster J, Ganatra M, Kamal I, Ware J, Makarova K, Ivanova N, Bhattacharyya A, Kapatral V, Kumar S, Posfai J, Vincze T, Ingram J, Moran L, Lapidus A, Omelchenko M, Kyrpides N, Ghedin E, Wang S, Goltsman E, Joukov V, Ostrovskaya O, Tsukerman K, Mazur M, Comb D, Koonin E, Slatko B (2005) The Wolbachia genome of Brugia malayi: endosymbiont evolution within a human pathogenic nematode. PLoS Biol 3: e121

Foster JM, Kumar S, Ford L, Johnston KL, Ben R, Graeff-Teixeira C, Taylor MJ (2008) Absence of Wolbachia endobacteria in the nonfilariid nematodes Angiostrongylus cantonensis and A. costaricensis. Parasit Vectors 1:31

Foster JM, Raverdy S, Ganatra MB, Colussi PA, Taron CH, Carlow CK (2009) The Wolbachia endosymbiont of Brugia malayi has an active phosphoglycerate mutase: a candidate target for antifilarial therapies. Parasitol Res 104:1047-1052

Ghedin E, Wang S, Spiro D, Caler E, Zhao Q, Crabtree J, Allen JE, Delcher AL, Guiliano DB, Miranda-Saavedra D, Angiuoli SV, Creasy T, Amedeo P, Haas B, El-Sayed NM, Wortman JR, Feldblyum T, Tallon L, Schatz M, Shumway M, Koo H, Salzberg SL, Schobel S, Pertea M, Pop M, White O, Barton GJ, Carlow CK, Crawford MJ, Daub J, Dimmic MW, Estes CF, Foster JM, Ganatra M, Gregory WF, Johnson NM, Jin J, Komuniecki R, Korf I, Kumar S, Laney S, Li BW, Li W, Lindblom TH, Lustigman S, Ma D, Maina CV, Martin DM, McCarter JP, McReynolds L, Mitreva M, Nutman TB, Parkinson J, PeregrinAlvarez JM, Poole C, Ren Q, Saunders L, Sluder AE, Smith K, Stanke M, Unnasch TR, Ware J, Wei AD, Weil G, Williams DJ, Zhang Y, Williams SA, Fraser-Liggett C, Slatko B, Blaxter ML, Scott AL (2007) Draft genome of the filarial nematode parasite Brugia malayi. Science 317:1756-1760

Ghedin E, Daehnel K, Foster J, Slatko B, Lustigman S (2008) The symbiotic relationship between filarial parasitic nematodes and their Wolbachia endosymbionts-A resource for a new generation of control measures. Symbiosis 46:77-85

Ghedin E, Hailemariam T, Depasse JV, Zhang X, Oksov Y, Unnasch TR, Lustigman S (2009) Brugia malayi gene expression in response to the targeting of the Wolbachia endosymbiont by tetracycline treatment. PLoS Negl Trop Dis 3:e525

Haegeman A, Vanholme B, Jacob J, Vandekerckhove TT, Claeys M, Borgonie G, Gheysen G (2009) An endosymbiotic bacterium in a plant-parasitic nematode: member of a new Wolbachia supergroup. Int J Parasitol 39:1045-1054 
Heath BD, Butcher RD, Whitfield WG, Hubbard SF (1999) Horizontal transfer of Wolbachia between phylogenetically distant insect species by a naturally occurring mechanism. Curr Biol 9:313-316

Hedges LM, Brownlie JC, O’Neill SL, Johnson KN (2008) Wolbachia and virus protection in insects. Science 322:702

Henrichfreise B, Schiefer A, Schneider T, Nzukou E, Poellinger C, Hoffmann TJ, Johnston KL, Moelleken K, Wiedemann I, Pfarr K, Hoerauf A, Sahl HG (2009) Functional conservation of the lipid II biosynthesis pathway in the cell wall-less bacteria Chlamydia and Wolbachia: why is lipid II needed? Mol Microbiol 73:913-923

Hoerauf A, Nissen-Pahle K, Schmetz C, Henkle-Duhrsen K, Blaxter ML, Buttner DW, Gallin MY, Al-Qaoud KM, Lucius R, Fleischer B (1999) Tetracycline therapy targets intracellular bacteria in the filarial nematode Litomosoides sigmodontis and results in filarial infertility. J Clin Invest 103:11-18

Hoerauf A, Mand S, Adjei O, Fleischer B, Buttner DW (2001) Depletion of Wolbachia endobacteria in Onchocerca volvulus by doxycycline and microfilaridermia after ivermectin treatment. Lancet 357:1415-1416

Hoerauf A, Specht S, Buttner M, Pfarr K, Mand S, Fimmers R, MarfoDebrekyei Y, Konadu P, Debrah AY, Bandi C, Brattig N, Albers A, Larbi J, Batsa L, Taylor MJ, Adjei O, Buttner DW (2008) Wolbachia endobacteria depletion by doxycycline as antifilarial therapy has macrofilaricidal activity in onchocerciasis: a randomized placebocontrolled study. Med Microbiol Immunol 197:295-311

Holman AG, Davis PJ, Foster JM, Carlow CK, Kumar S (2009) Computational prediction of essential genes in an unculturable endosymbiotic bacterium, Wolbachia of Brugia malayi. BMC Microbiol 9:243

Hosokawa T, Koga R, Kikuchi Y, Meng XY, Fukatsu T (2010) Wolbachia as a bacteriocyte-associated nutritional mutualist. Proc Natl Acad Sci U S A 107:769-774

Hotopp JC, Clark ME, Oliveira DC, Foster JM, Fischer P, Torres MC, Giebel JD, Kumar N, Ishmael N, Wang S, Ingram J, Nene RV, Shepard J, Tomkins J, Richards S, Spiro DJ, Ghedin E, Slatko BE, Tettelin H, Werren JH (2007) Widespread lateral gene transfer from intracellular bacteria to multicellular eukaryotes. Science 317:1753-1756

Ishmael N, Hotopp JC, Ioannidis P, Biber S, Sakamoto J, Siozios S, Nene V, Werren J, Bourtzis K, Bordenstein SR, Tettelin H (2009) Extensive genomic diversity of closely related Wolbachia strains. Microbiology 155:2211-2222

Jacob J, Mitreva M, Vanholme B, Gheysen G (2008) Exploring the transcriptome of the burrowing nematode Radopholus similis. Mol Genet Genomics 280:1-17

Juhas M, Crook DW, Hood DW (2008) Type IV secretion systems: tools of bacterial horizontal gene transfer and virulence. Cell Microbiol 10:2377-2386

Keiser PB, Coulibaly Y, Kubofcik J, Diallo AA, Klion AD, Traore SF, Nutman TB (2008) Molecular identification of Wolbachia from the filarial nematode Mansonella perstans. Mol Biochem Parasitol 160:123-128

Kozek WJ (1977) Transovarially-transmitted intracellular microorganisms in adult and larval stages of Brugia malayi. J Parasitol 63:992-1000

Kozek WJ, Marroquin HF (1977) Intracytoplasmic bacteria in Onchocerca volvulus. Am J Trop Med Hyg 26:663-678

Lin M, den Dulk-Ras A, Hooykaas PJ, Rikihisa Y (2007) Anaplasma phagocytophilum AnkA secreted by type IV secretion system is tyrosine phosphorylated by Abl-1 to facilitate infection. Cell Microbiol 9:2644-2657

Lo N, Casiraghi M, Salati E, Bazzocchi C, Bandi C (2002) How many Wolbachia supergroups exist? Mol Biol Evol 19:341-346

Lo N, Paraskevopoulos C, Bourtzis K, O'Neill SL, Werren JH, Bordenstein SR, Bandi C (2007) Taxonomic status of the intracellular bacterium Wolbachia pipientis. Int J Syst Evol Microbiol 57:654-657
Mand S, Pfarr K, Sahoo PK, Satapathy AK, Specht S, Klarmann U, Debrah AY, Ravindran B, Hoerauf A (2009) Macrofilaricidal activity and amelioration of lymphatic pathology in bancroftian filariasis after 3 weeks of doxycycline followed by single-dose diethylcarbamazine. Am J Trop Med Hyg 81:702-711

McGarry HF, Egerton GL, Taylor MJ (2004) Population dynamics of Wolbachia bacterial endosymbionts in Brugia malayi. Mol Biochem Parasitol 135:57-67

McLaren DJ, Worms MJ, Laurence BR, Simpson MG (1975) Microorganisms in filarial larvae (Nematoda). Trans R Soc Trop Med Hyg 69:509-514

McMeniman CJ, Lane RV, Cass BN, Fong AW, Sidhu M, Wang YF, O'Neill SL (2009) Stable introduction of a life-shortening Wolbachia infection into the mosquito Aedes aegypti. Science 323:141-144

McNulty SN, Foster JM, Mitreva M, Dunning Hotopp JC, Martin J, Fischer K, Wu B, Davis PJ, Kumar S, Brattig NW, Slatko BE, Weil GJ, Fischer PU (2010) Endosymbiont DNA in endobacteria-free filarial nematodes indicates ancient horizontal gene transfer. Plos ONE (in press)

Molyneux DH, Bradley M, Hoerauf A, Kyelem D, Taylor MJ (2003) Mass drug treatment for lymphatic filariasis and onchocerciasis. Trends Parasitol 19:516-522

Moran NA, Plague GR, Sandstrom JP, Wilcox JL (2003) A genomic perspective on nutrient provisioning by bacterial symbionts of insects. Proc Natl Acad Sci U S A 100(Suppl 2):14543-14548

Moreira LA, Iturbe-Ormaetxe I, Jeffery JA, Lu G, Pyke AT, Hedges LM, Rocha BC, Hall-Mendelin S, Day A, Riegler M, Hugo LE, Johnson KN, Kay BH, McGraw EA, van den Hurk AF, Ryan PA, O'Neill SL (2009) A Wolbachia symbiont in Aedes aegypti limits infection with dengue, Chikungunya, and Plasmodium. Cell 139:1268-1278

Muller R (2002) Worms and human disease. CABI Publishing, Wallingford

Osei-Atweneboana MY, Eng JK, Boakye DA, Gyapong JO, Prichard RK (2007) Prevalence and intensity of Onchocerca volvulus infection and efficacy of ivermectin in endemic communities in Ghana: a two-phase epidemiological study. Lancet 369:20212029

Pan X, Luhrmann A, Satoh A, Laskowski-Arce MA, Roy CR (2008) Ankyrin repeat proteins comprise a diverse family of bacterial type IV effectors. Science 320:1651-1654

Pannebakker BA, Loppin B, Elemans CP, Humblot L, Vavre F (2007) Parasitic inhibition of cell death facilitates symbiosis. Proc Natl Acad Sci U S A 104:213-215

Panteleev D, Goriacheva II, Andrianov BV, Reznik NL, Lazebnyi OE, Kulikov AM (2007) The endosymbiotic bacterium Wolbachia enhances the nonspecific resistance to insect pathogens and alters behavior of Drosophila melanogaster. Genetika 43:1277-1280

Pfarr K, Foster J, Slatko B (2007a) It takes two: lessons from the first nematode Wolbachia genome sequence. In: Hoerauf A, Rao RU (eds) Wolbachia: a bug's life in another bug. Karger, Basel, pp $52-65$

Pfarr K, Foster J, Slatko B, Hoerauf A, Eisen JA (2007b) On the taxonomic status of the intracellular bacterium Wolbachia pipientis: should this species name include the intracellular bacteria of filarial nematodes? Int J Syst Evol Microbiol $57: 1677-1678$

Podlaha O, Zhang J (2009) Processed pseudogenes: the 'fossilized footprints' of past gene expression. Trends Genet 25:429-434

Rao AU, Carta LK, Lesuisse E, Hamza I (2005) Lack of heme synthesis in a free-living eukaryote. Proc Natl Acad Sci U S A 102:4270-4275

Raverdy S, Foster JM, Roopenian E, Carlow CK (2008) The Wolbachia endosymbiont of Brugia malayi has an active pyruvate phosphate dikinase. Mol Biochem Parasitol 160:163166 
Shigenobu S, Watanabe H, Hattori M, Sakaki Y, Ishikawa H (2000) Genome sequence of the endocellular bacterial symbiont of aphids Buchnera sp. APS. Nature 407:81-86

Sinkins SP, Godfray HC (2004) Use of Wolbachia to drive nuclear transgenes through insect populations. Proc Biol Sci 271:14211426

Sinkins SP, Gould F (2006) Gene drive systems for insect disease vectors. Nat Rev Genet 7:427-435

Sironi M, Bandi C, Sacchi L, Di Sacco B, Damiani G, Genchi C (1995) Molecular evidence for a close relative of the arthropod endosymbiont Wolbachia in a filarial worm. Mol Biochem Parasitol 74:223-227

Specht S, Mand S, Marfo-Debrekyei Y, Debrah AY, Konadu P, Adjei O, Buttner DW, Hoerauf A (2008) Efficacy of 2- and 4-week rifampicin treatment on the Wolbachia of Onchocerca volvulus. Parasitol Res 103:1303-1309

Strubing U, Lucius R, Hoerauf A, Pfarr KM (2010) Mitochondrial genes for heme-dependent respiratrory chain complexes are upregulated after depletion of Wolbachia from filarial nematodes. Int J Parasitol

Sun LV, Foster JM, Tzertzinis G, Ono M, Bandi C, Slatko BE, O’Neill SL (2001) Determination of Wolbachia genome size by pulsedfield gel electrophoresis. J Bacteriol 183:2219-2225

Supali T, Djuardi Y, Pfarr KM, Wibowo H, Taylor MJ, Hoerauf A, Houwing-Duistermaat JJ, Yazdanbakhsh M, Sartono E (2008) Doxycycline treatment of Brugia malayi-infected persons reduces microfilaremia and adverse reactions after diethylcarbamazine and albendazole treatment. Clin Infect Dis 46:13851393

Taylor MJ (2002) Wolbachia bacterial endosymbionts. In: Klei TR, Rajan TV (eds), The filaria. Kluwer Academic Publishers, pp 143-153

Taylor MJ, Bandi C, Hoerauf A (2005) Wolbachia bacterial endosymbionts of filarial nematodes. Adv Parasitol 60:245-284
Telschow A, Hammerstein P, Werren JH (2005) The effect of Wolbachia versus genetic incompatibilities on reinforcement and speciation. Evolution 59:1607-1619

Tsai KH, Huang CG, Wang LC, Yu YW, Wu WJ, Chen WJ (2007) Molecular evidence for the endosymbiont Wolbachia in a nonfilaroid nematode, Angiostrongylus cantonensis. J Biomed Sci 14:607-615

Turner JD, Tendongfor N, Esum M, Johnston KL, Langley RS, Ford L, Faragher B, Specht S, Mand S, Hoerauf A, Enyong P, Wanji S, Taylor MJ (2010) Macrofilaricidal activity after doxycycline only treatment of Onchocerca volvulus in an area of Loa loa coendemicity: a randomized controlled trial. PLoS Negl Trop Dis 4: e660

Wanji S, Tendongfor N, Nji T, Esum M, Che JN, Nkwescheu A, Alassa F, Kamnang G, Enyong PA, Taylor MJ, Hoerauf A, Taylor DW (2009) Community-directed delivery of doxycycline for the treatment of onchocerciasis in areas of co-endemicity with loiasis in Cameroon. Parasit Vectors 2:39

Werren JH (1997) Biology of Wolbachia. Annu Rev Entomol 42:587609

Werren JH, Baldo L, Clark ME (2008) Wolbachia: master manipulators of invertebrate biology. Nat Rev Microbiol 6:741-751

Williams SA, Lizotte-Waniewski MR, Foster J, Guiliano D, Daub J, Scott AL, Slatko B, Blaxter ML (2000) The filarial genome project: analysis of the nuclear, mitochondrial and endosymbiont genomes of Brugia malayi. Int J Parasitol 30:411-419

Wu D, Daugherty SC, Van Aken SE, Pai GH, Watkins KL, Khouri H, Tallon LJ, Zaborsky JM, Dunbar HE, Tran PL, Moran NA, Eisen JA (2006) Metabolic complementarity and genomics of the dual bacterial symbiosis of sharpshooters. PLoS Biol 4:e188

Wu B, Novelli J, Foster J, Vaisvila R, Conway L, Ingram J, Ganatra M, Rao AU, Hamza I, Slatko B (2009) The heme biosynthetic pathway of the obligate Wolbachia endosymbiont of Brugia malayi as a potential anti-filarial drug target. PLoS Negl Trop Dis 3:e475 